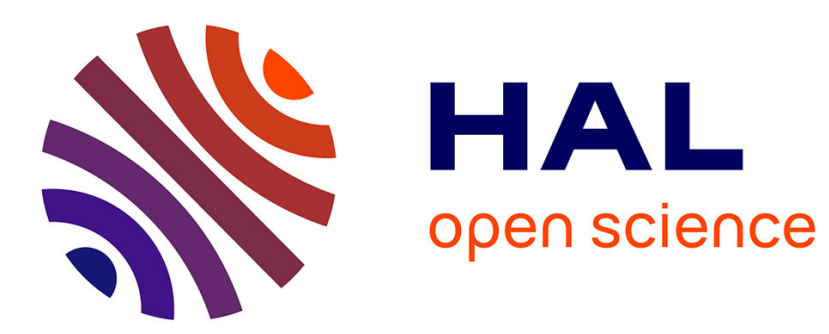

\title{
The sound production mechanism associated with coherent structures in subsonic jets
}

\author{
A. Guitton, F. Kerherve, P. Jordan, J. Delville
}

\section{To cite this version:}

A. Guitton, F. Kerherve, P. Jordan, J. Delville. The sound production mechanism associated with coherent structures in subsonic jets. 14th AIAA/CEAS Aeroacoustics Conference (29th AIAA Aeroacoustics Conference), May 2008, Vancouver, Canada. 10.2514/6.2008-2892 . hal-00403611

\section{HAL Id: hal-00403611 \\ https://hal.science/hal-00403611}

Submitted on 18 Feb 2022

HAL is a multi-disciplinary open access archive for the deposit and dissemination of scientific research documents, whether they are published or not. The documents may come from teaching and research institutions in France or abroad, or from public or private research centers.
L'archive ouverte pluridisciplinaire HAL, est destinée au dépôt et à la diffusion de documents scientifiques de niveau recherche, publiés ou non, émanant des établissements d'enseignement et de recherche français ou étrangers, des laboratoires publics ou privés.

\section{다)(1) $(5$}

Distributed under a Creative Commons Attribution - NonCommerciall 4.0 International 


\title{
The sound production mechanism associated with coherent structures in subsonic jets
}

\author{
A. Guitton, F. Kerhervé, P. Jordan* \& J. Delville*
}

\begin{abstract}
In this work we address the question regarding the mechanism by which coherent structures in high Reynolds number jets generate sound. Previous work by Coiffet et al. ${ }^{7}$ and Guitton et al. ${ }^{16}$ has provided evidence that the mechanism may correspond to a linear, wavy-wall scenario - coherence nodes observed in nearfield measurements, and thought to correspond to destructive interference between convecting hydrodynamic perturbations and propagating sound waves, were interpreted as the signature of such a mechanism.

We investigate this hypothesis by means of a simple model problem comprising an axisymmetric, subsonically-convecting wavepacket structure, with Gaussian axial and radial envelopes. The model reproduces the interference nodes observed experimentally.

We then compare the results with experimental data acquired in the nearfield of a subsonic jet $\left(M=0.6, R e=1.10^{6}\right)$. This data is filtered to separate hydrodynamic and acoustic nearfield signatures, such that quantitative comparison is possible between the sound field generated by the model and that observed experimentally. Results show...
\end{abstract}

\section{Introduction}

The role played by so-called coherent structures in the production of sound by high Reynolds-number turbulent jets has been the subject of considerable attention since the first experimental observation of such structures in free shear-flows. In the 1970s and 1980s experiments confirmed that the sound field radiated by subsonic jets is dominated by low-order azimuthal Fourier modes (Michalke and Fuchs ${ }^{32}$; Maestrello ${ }^{29}$; Fuchs and Michel $^{14}$; Juvé et al. ${ }^{19}$ ), and similar conclusions were later drawn regarding the azimuthal organisation of the turbulent jet (Fuchs ${ }^{14}$; Amrstrong et al. ${ }^{2}$ ). However, because modal decompositions are less straightforward in the axial and radial directions - both because it is not possible to place large numbers of axially-distributed measurement probes in the turbulent region of the flow, and because Fourier decompositions are not well-defined in these inhomogeneous directions - it is not so easy to assess the axial and radial organisation of the jet in the same manner. Pressure measurements performed in the irrotational nearfield using axially distrbuted microphones constitute an interesting alternative to in-flow measurements, as the flow contamination problem is thereby precluded. This kind of measurement approach has been implemented in subsonic and supersonic flows for nearly fifty years now (Mayes ${ }^{31}$; Howes ${ }^{18}$; Mollo-Christensen ${ }^{34}$; Keast \& Maidanik ${ }^{21}$; Ollerhead $^{38}$; Arndt et al. ${ }^{3}$; Ricaud $^{41}$; Reba et al. ${ }^{40}$; Coiffet et al. ${ }^{7}$; Barre et al. ${ }^{4}$; Suzuki \& Colonius ${ }^{47}$ ) and the characteristic of the nearfield which all investigators have found most striking is its degree of axial organisation. In order to investigate the sound production mechanisms which might be associated with such organised flow-patterns, many studies have been performed where acoustic excitation is used to enhance the degree of organisation of the flow (Moore ${ }^{35}$; Kibens ${ }^{23}$; Laufer \& Yen ${ }^{27}$; Arbey \& Ffowcs-Williams ${ }^{1}$; Bridges \& Hussain ${ }^{5}$; Ghosh et al. ${ }^{15}$; Fleury et al. ${ }^{13}$ ). Such deterministic space-time structures are amenable to analytical modelling (Ffowcs-Williams \& Kempton ${ }^{12}$ ; Mankbadi \& Liu ${ }^{30}$; Crighton \& Huerre ${ }^{9}$; Sandham \& Morfey $^{42}$; Kopiev \& Chernyshev ${ }^{26}$ ). And, low Reynolds number flows dominated by coherent vortex dynamics were the first to be studied numerically (Mitchell et al. ${ }^{33}$; Colonius et al. ${ }^{8}$ ).

\section{A. Instability wave or coherent structure ?}

The nearfield of the turbulent jet constitutes a complex flow-regime however, difficult to interpret, and while the signature it comprises strongly resembles linear hydrodynamic instability waves, there is little doubt

\footnotetext{
${ }^{*}$ Laboratoire d'Etudes Aérodynamiques, CNRS UMR 6609, Université de Poitiers, ENSMA, France.
} 


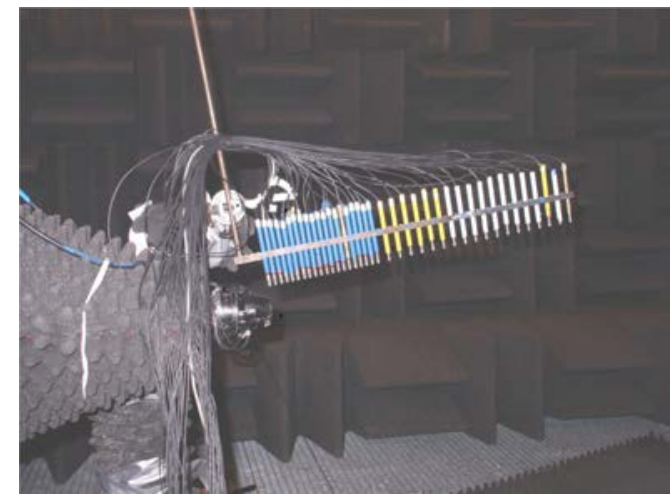

(a)

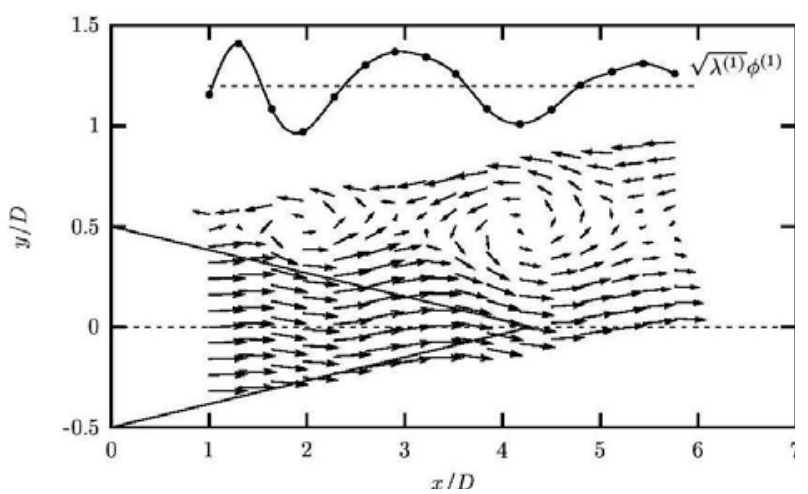

(b)

Figure 1. (a) Experimenal setup of current work; (b) Reconstruction of the instantaneous velocity in a low Mach number jet by linear stochastic estimation using microphone measurements along the outer edge of the mixing layer; taken from. ${ }^{39}$

that it is driven by intense vortical motion in the shear-layer(s). Chan ${ }^{6}$ was first to perform quantitative comparisons between nearfield signatures and linear instability theory; Suzuki and Colonius ${ }^{47}$ have more recently confirmed that the signatures can be thought of as synonymous with linear instabilities; Picard and Delville ${ }^{39}$ have shown that these nearfield fluctuations correspond to, and are linearly driven by, large-scale, organised vortical mixing-layer structures (see figure 1) in a low Mach number flow, while Tinney et al. ${ }^{49}$ have reproduced this result in a high Mach number, high Reynolds-number flow.

\section{B. Source mechanisms}

Despite this considerable body of work, there is no general consensus regarding the mechanisms by which coherent structures generate sound; some scientists still doubt their importance; and, where they are admitted, it is not clear what contribution they make to the sound power radiated by high Reynolds number flows. A number of conflicting views are currently held regarding the 'coherent' sound-production mechanism. The linear wavy-wall model is frequently argued to be too weak a generator of sound to be important in flows with subsonic convection velocities. However, amplification and decay (Crighton \& Huerre ${ }^{9}$; Ffowcs-Williams \& Kempton ${ }^{12}$ ), space-time randomness in the phase $\left(\right.$ Moore $^{35}$ ) and amplitude modulation (Morfey et al. ${ }^{37}$ ) of wavepackets can enhance the radiation efficiency of such linear mechanisms. Non-linear interactions have been proposed by Sandham et al. ${ }^{42}$ as another means by which linear instability waves can become efficient sound generators. Vortex eigenmodes have been proposed by Kopiev et al. ${ }^{24}$ as a possible mechanism, and vortex-pairing is also believed to be important (Ffowcs-Williams \& Kempton ${ }^{12}$ ).

Experimental and numerical evidence is available which supports the existence of all of these mechanisms, showing, either that all of the said mechanisms play a role in the production of jet-noise, or, that we are often very good at finding whatever it is we set out to find! The vortex-pairing and quadratic non-linear interaction scenarios find support in excited and low-Reynolds number flows (Sandham et al. ${ }^{42}$; Laufer \& Yen $^{27}$; Morrison \& McLaughlin $\left.{ }^{36}\right)$. In higher Reynolds number flows there is considerable evidence for a linear mechanism (Lee \& Ribner ${ }^{28}$; Siddon \& Rackl ${ }^{46}$; Sharton \& White ${ }^{44}$; Seiner ${ }^{45}$; Juvé et al ${ }^{20}$; Schaffar $\& \mathrm{Hancy}^{43}$ ) the precise details of which are not clear. And, acoustic signatures similar to those generated by the octupole vortex eigenmode model can also be shown to exist (Kopiev \& Chernyshev $^{26}$; Kopiev et al. $\left.{ }^{25}\right)$.

\section{Contribution of the present paper}

In all of the above cases, support for a given model is provided by a demonstration of agreement between the farfield predicted by a model and that measured in an experiment. However, on account of the non-uniqueness problem, such demonstrations are always open to criticism: there are an infinity of source structures which can produce any given farfield. In this work we use nearfield pressure measurements with a view to mitigating 
this uncertainty. The measurements are performed in a region where both hydrodynamic and acoustic fluctuations are present, and where we thus sense both cause and effect at the same space-time coordinates.

Coiffet et al. ${ }^{7}$ observed an interference mechanism between the hydrodynamic and acoustic components of the pressure field in this region of the flow. A simple model was proposed to explain the interference phenomenon, and this agreed well with experimental observations: when the hydrodynamic and acoustic components of the pressure field have similar energy and are out of phase, localised cancellation of the coherence is observed. This led to the conjecture that the coherent structures in the initial mixing-layer region generate sound by means of a quasi-irrotational, linear source mechanism. The result is an important one, because it constitutes a more direct illustration of the sound-producing dynamics. As interpreted by Coiffet et al., ${ }^{7}$ we see in the interference mechanism the subtle motions implicated in the imbalance between reactive and resistive energy-fluxes which occurs during the production of propagative fluctuation-energy, i.e. the essence of a sound-source mechanism.

Recent analytical work by Le Dizès and Millet ${ }^{11}$ has demonstrated the existence of localised pressure minima where inner and outer solutions of a transonic instability wave problem coexist and interfere destructively. Their result is similar to that presented herein, except that in our work we consider the three dimensional problem at lower Mach number.

In what follows, after briefly describing the experiment, we recall the result and its interpretation; we then use a simple model to demonstrate that such interference patterns do in fact occur when sound is generated by a linear, wavy-volume source-mechanism in a potential flow. Finally, we extract bandpassfiltered hydrodynamic and acoustic signatures from the experimental nearfield data, and we compare these with the signatures produced by the linear, monochromatic model.

\section{Experimental set-up}

For details of the experiment the reader can refer to Coiffet, ${ }^{7}$ and similar results have been reported by Guitton et al ${ }^{16,17}$ for experiments performed under fully anechoic conditions and for a larger range of Mach numbers and axial positions. The experimental setup is shown in figure 1: a line array of microphones is positioned in the irrotational region of the jet, where both hydrodynamic and acoustic signatures are sensed.

\section{The interference mechanism}

The aforesaid interference mechanism was discovered by Coiffet et al. ${ }^{7}$ as a result of nulls which can be observed in the nearfield space-frequency coherence patterns. These nulls are seen at the intersection of the green and red lines in figure 2; with respect to a given reference microphone, there exist precise space-frequency coordinates at which the coherence suddenly drops to zero.

Such nulls can only be explained by one of three scenarios. Either the pressure fluctuation at the said space-frequency coordinate is: (1) zero; (2) linearly unrelated to the corresponding spectral component at the reference microphone; or, (3) comprises an interference between two highly correlated, but distinct components, which at that point have equal energy and opposite phase and thus cancel one another by means of destructive interference. The first of these possibilities is ruled out by the pressure spectrum, which has no discontinuity at the space-frequency coordinates of the nulls. The second scenario is highly improbable given the otherwise continuous shape of the space-frequency coherence patterns - such an hypothesis would imply that a very narrow band of spectral components are linearly decoupled from fluctuations of the same frequency at the reference microphone, whereas outside of that narrow band of frequencies relatively strong linear relationships exist. This leaves us with the third scenario, which is that proposed by Coiffet et al. ${ }^{7}$ The reactive and propagative components of the nearfield are considered to correspond, respectively, to the coherent structures (or deterministic instabilities) and the sound field they radiate; they are considered to be highly correlated and dephased by $\pi / 2$ (implying a linear, irrotational source-mechanism); the reactive component is characterised by axial convection velocities and very rapid radial decay (in keeping with the evanescent character of reactive acoustic pressure fluctuations), whereas the acoustic component radiates spherically at the speed of sound, and decays much more slowly $(1 / r)$. With these assumptions, energy and phase criteria can be easily identified where, with respect to some reference point, the two fields will, at some localised space-frequency coordinate, have equal energy and opposite phase, and will thus interfere destructively to completely remove all pressure fluctuations coherent with the corresponding spectral components 

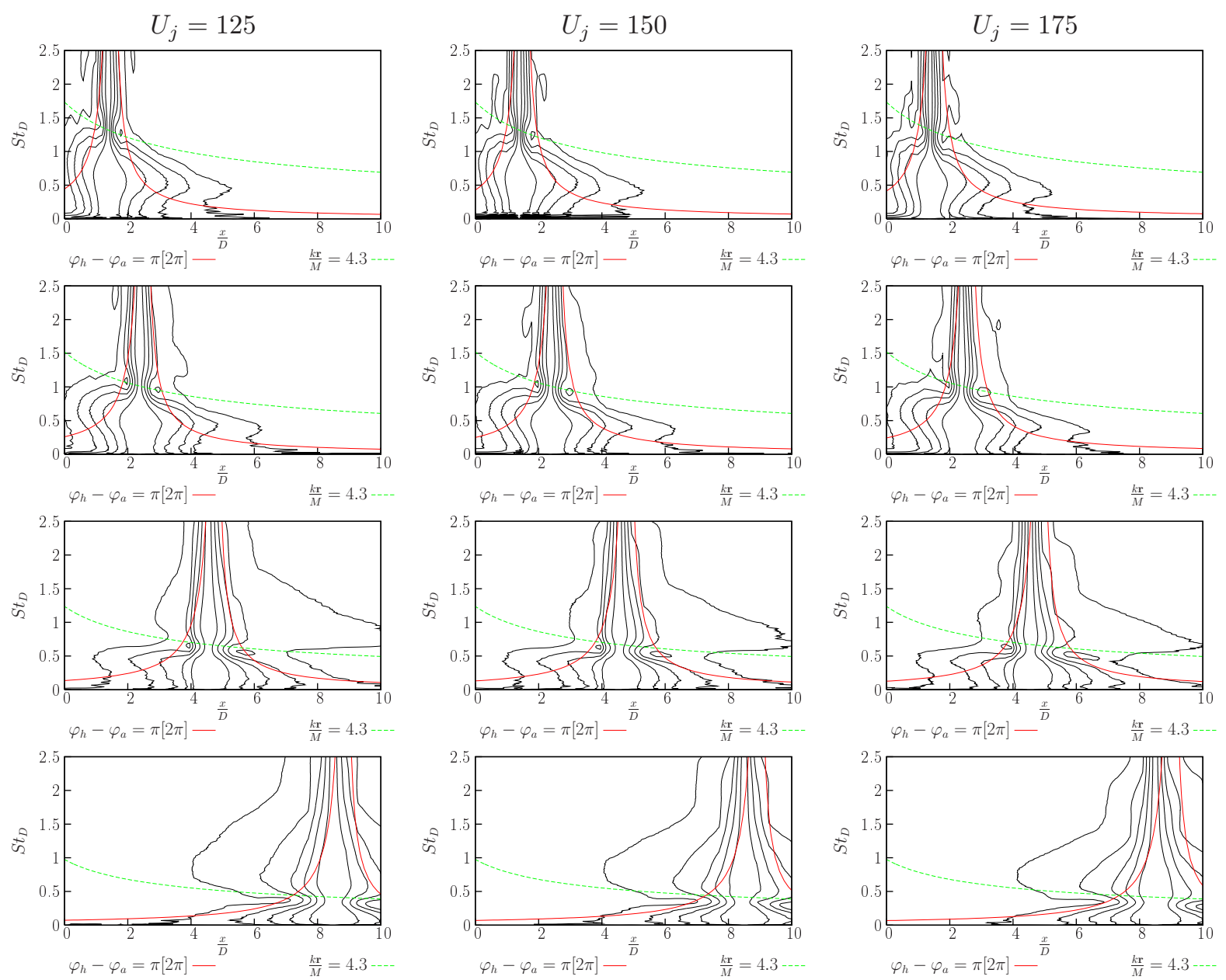

Figure 2. Coherence and interference criteria in space-frequency domain. Isocontours of coherence (solid black line); space-frequency coordinates where hydrodynamic and acoustic fluctuations have equal energy (green line); space-frequency coordinates where hydrodynamic and acoustic fluctuations are dephased by ( $\pi$ ) (red lines). Nulls are observed in regions where the two criteria are satisfied (intersection of the red and green lines).

at the reference position. These criteria are identified by the red and green lines shown in figure 2, and as can be seen from the figure, their intersection (the points at which both criteria are satisfied) agree well with the observed nulls for a considerable range of axial position and exit velocities.

\section{An analytical model}

We now turn our attention to a simplified model problem in order to investigate if such interference can really be produced by a sound production mechanism comprising a linear, volume source in a potential flow. This model shares some similarities with those of Ffowcs-Williams and Kempton, ${ }^{12}$ Crighton and Huerre ${ }^{9}$ and Morfey et al., ${ }^{37}$ but has two important differences: (1) we consider a volume distribution rather than the line distributions of the said work; and, (2) we study the nearfield solution rather than that of the farfield, in order to compare with our experiments, but also for the reasons evoked earlier regarding the non-uniqueness of farfield solutions.

The source distribution is modelled as:

$$
\begin{aligned}
q(\boldsymbol{x}, t) & =q(x, r, \theta, t)=\Delta \phi_{s}(\boldsymbol{x}, t) \\
& =e^{-\frac{\left(x-x_{0}\right)^{2}}{\lambda_{x}^{2}}-\frac{\left(r-r_{0}\right)^{2}}{\lambda_{r}^{2}}} \cos \left(2 \pi\left(k_{x} x-U_{c} k_{x} t\right)\right) \cos m \theta
\end{aligned}
$$


where $\phi_{s}$ is the imposed source potential, $U_{c}$ is a typical convection velocity. $x, y \& z$ are cartesian volume coordinates, which can be directly associated with the cylindrical coordinates $x, r \& \theta$ by the relationships $x=x, y=r \cos \theta \& z=r \sin \theta$. The superscript ${ }^{*}$ implies dimensionless quantities; dimensions are normalised by D, where $\mathrm{D}$ is the diameter of the ring-like structure of the excitation (synonymous with the exit diameter of a jet). We only consider the axisymmetric mode in this work $(m=0)$; however, higher-order azimuthal modes can be easily included. $\lambda_{x}$ and $\lambda_{r}$ define the axial and radial scales of the wave envelope. The gaussian amplitude modulation is chosen to mimic the amplification-to-decay behaviour of instabilities in the upstream region of a jet. $k_{x}$ is the axial wavenumber and it represents the spatial structure of the wave in the convective direction. In the present paper, the following values have been chosen for the variables: $U_{c}=0.5 c$ (where $c$ is the speed of sound), $k_{x} D=1.0, \lambda_{x}=2.0 D, \lambda_{r}=0.5 D, x_{0}=1.0 D$. A snapshot of the source-field is shown in figure $3(\mathrm{a})$.

The sound production problem considered is governed by the equation:

$$
\square^{2} \phi_{a}(\boldsymbol{x}, t)=q(\boldsymbol{x}, t),
$$

where $\phi_{a}$ is the acoustic potential which results from the excitation; and we study the integral, retarded-

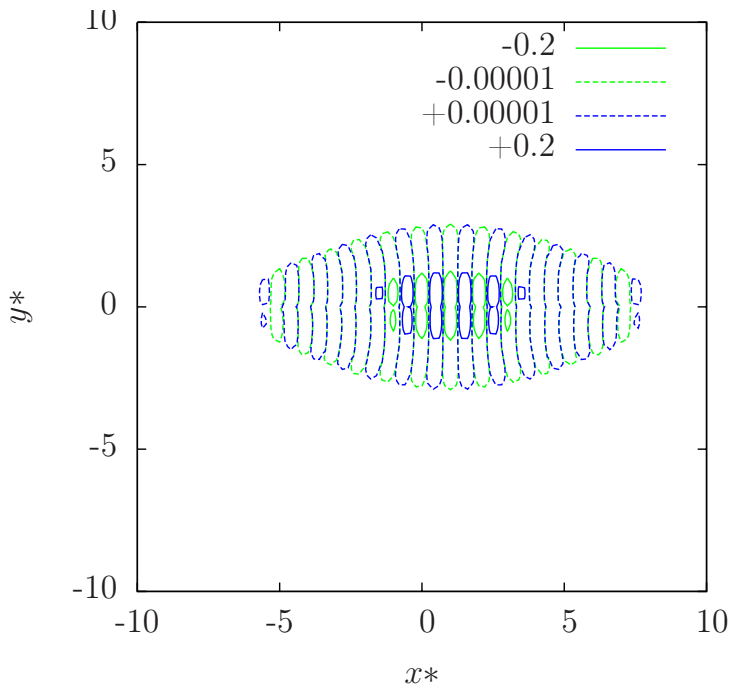

(a)

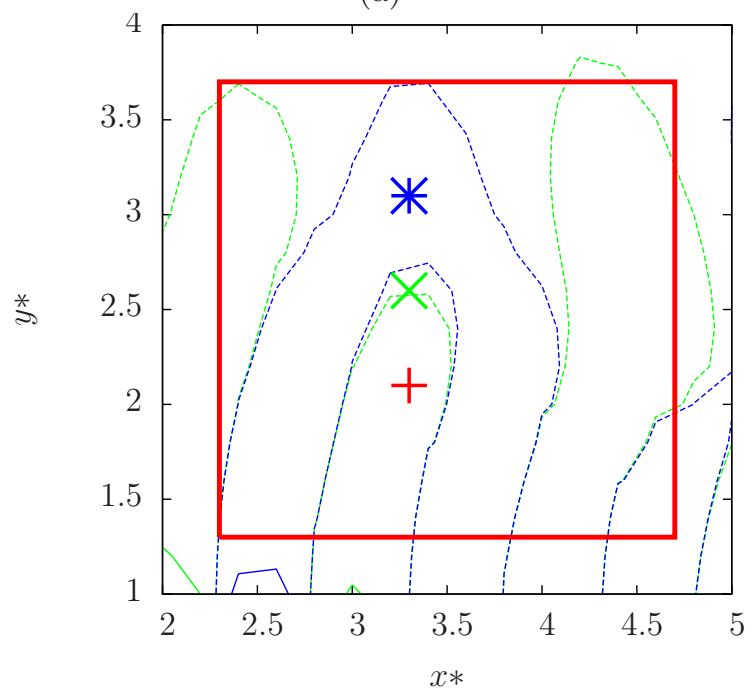

(c)

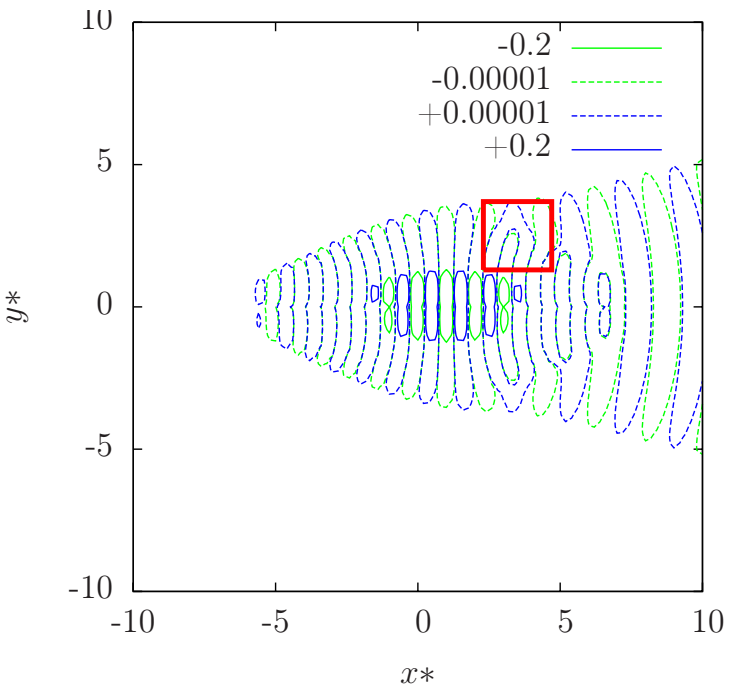

(b)

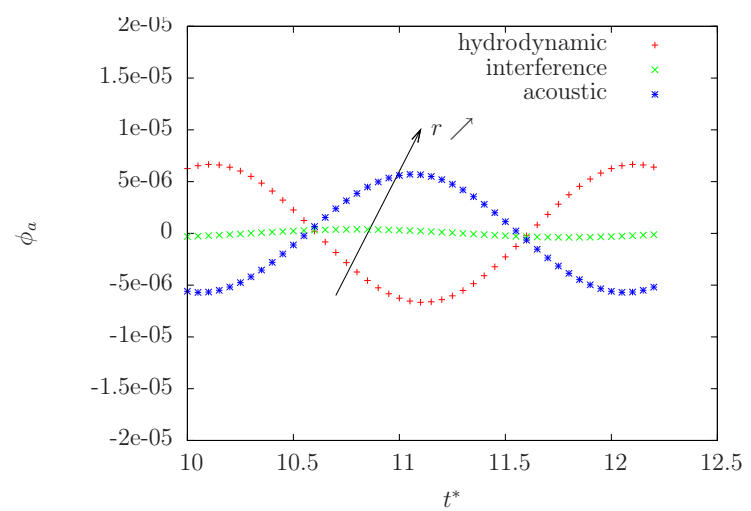

(d)

Figure 3. (a) Source potential; (b) nearfield acoustic-response (potential); (c) zoom on interference zone; (d) time histories of fluctuations at the points shown in (c): red: reactive; blue: propagative; green: the node resulting from their destructive interference (green) 
potential solution, obtained using the free space Greens function:

$$
\phi_{a}=\frac{1}{4 \pi} \int_{V} \frac{q\left(\boldsymbol{y}, t-\frac{|\boldsymbol{x}-\boldsymbol{y}|}{c}\right)}{|\boldsymbol{x}-\boldsymbol{y}|} d \boldsymbol{y} .
$$

As analytical solution is not possible in the nearfield, the integration is performed numerically. Two different grids are used. One corresponds to the points where the solution is evaluated $\left(\phi_{a}\right)$; this is a cartesian grid composed of $N_{y} * N_{z} * N_{x}$ points, which is adapted depending on the observation area and the grid density necessary to visualise the phenomena of interest. For example, to obtain the field from figure $3(\mathrm{c}), 2^{*} 100^{*} 100$ points were necessary $\left(\frac{x}{D}=0 . .1, \frac{y}{D}=-10 . .10\right.$ and $\left.\frac{z}{D}=-10 . .10\right)$ in order to resolve the interference phenomenon. The second cylindrical grid, defined by $N_{r} * N_{\theta} * N_{x}$, is used for the integration (as the source is specified in cylindrical coordinates). ${ }^{\mathrm{a}}$ In the present case $150^{*} 36^{*} 250$ points define the integration mesh from $\frac{r}{D}=0 . .3, \theta=0 . .2 \pi$ and $\frac{x}{D}=-0 . .15$.

The fields $\phi_{s}$ and $\phi_{a}$ are shown in figure $3(\mathrm{a})$ and (b). The interference mechanism discussed in the last section is found to occur at the coordinates $\left(x^{*}=3.37, y^{*}=2.64\right)$, shown in figures $3(\mathrm{~b})$ and (c) by the red square. The reactive and acoustic components - which can be clearly seen in figure $3(\mathrm{~b})$ in the two disparate spatial scales - are characterised by similar frequencies, but different phase-velocities (respectively $U_{c}$ and $c$ ). They consequently interfere destructively at the said. This is shown again in figure $3(\mathrm{~d})$, where the opposition of phase between the reactive and propagative components is manifest in the fluctuations at points just above and below the interference coordinates.

The mechanism proposed by Coiffet et al. ${ }^{7}$ is thus corroborated by this model problem. In the next section we will use the nearfield data to perform a more quantitative comparison, and in order to understand what contribution this mechanism makes to the farfield.

\section{Comparison with experimental nearfield data}

A frequency-wavenumber spectrum is computed from the nearfield data; this shown in figure 4(a) for an exit velocity of $200 \mathrm{~m} / \mathrm{s}$. Two lobes are observed, corresponding, respectively, to the convective and propagative signatures. We would like to examine how these signatures compare with the convective and propagating components observed using an analytical model such as that presented in the previous section. As the model only considers a single frequency, we extract the wavenumber spectrum for single-frequency components, and filter these in order to isolate hydrodynamic and acoustic perturbations. These are characterised, respectively, by subsonic and supersonic phase velocity, and so band-pass filters which pass either $\omega>k c$ or $\omega<k c$ can be used to remove one or the other of these components. A similar procedure is discussed and implemented in a companion paper $^{22}$

The result is shown in figure 4(b)-(f) for the five frequencies indicated by the solid black lines in figure 4(a). The hydrodynamic and acoustic energy distributions are shown in pink and red, respectively. These figures are in qualitative agreement with the wavy-wall model, insofar as we see hydrodynamic energy distribution (which can be thought of as synonymous with the wave-packet source) which always peaks just upstream of the acoustic energy-distribution.

The energy levels shown in figure 4 are representative of the signatures as measured on the array. However, as we have modelled it, the maximum source energy is to be found on the shear-layer axis. We must therefore extrapolate the measured data to its value within the jet. In order to do so we use the analytical form which describes the radial decay of an instability wave, $r^{-1} \exp \left(-r \sqrt{k^{2}-\omega^{2} / c^{2}}\right)$, as per Suzuki \& Colonius ${ }^{47}$ for example. The result is shown in figure 5 for the frequencies considered. The ratio of hydrodynamic-toacoustic energy is generally of the order of two to three orders of magnitude. This is a good deal less than the energy ratios observed in the linear model presented in the previous section. However, the said model was used only to see if the hypothetical interference mechanism could occur in such a wavy-wall scenario. The parameters used for the wave-envelope were very different from those characteristic of the jet. In the next phase of work, which is currently underway, wave-packet parameters are chosen to closely match the experimental data for the frequencies considered above, in order to obtain a quantitative comparison. This procedure works as follows. The hydrodynamic portion of the wavenumber spectrum is inverse Fourier transformed to obtain a spatial correlation curve; this provides a characteristic shape for the wave-envelope.

\footnotetext{
${ }^{a}$ We perform the solution on a cartesian grid on account of a subsequent study which we intend to perform using the formulations of $;{ }^{10}$ they propose cartesian tensor quantities for a more refined description of instantaneous, reactive and propagative, energy-fluxes.
} 


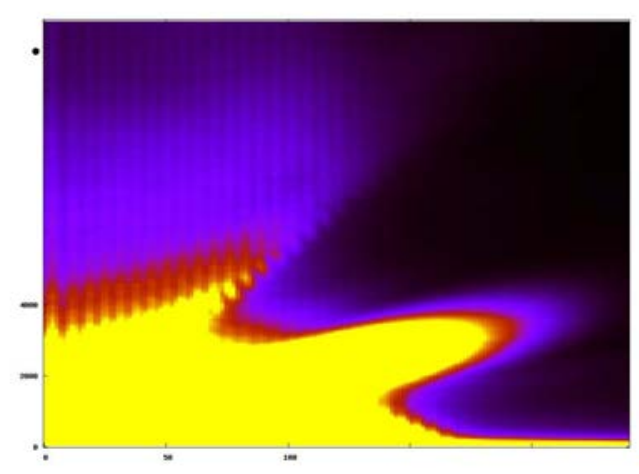

(a)

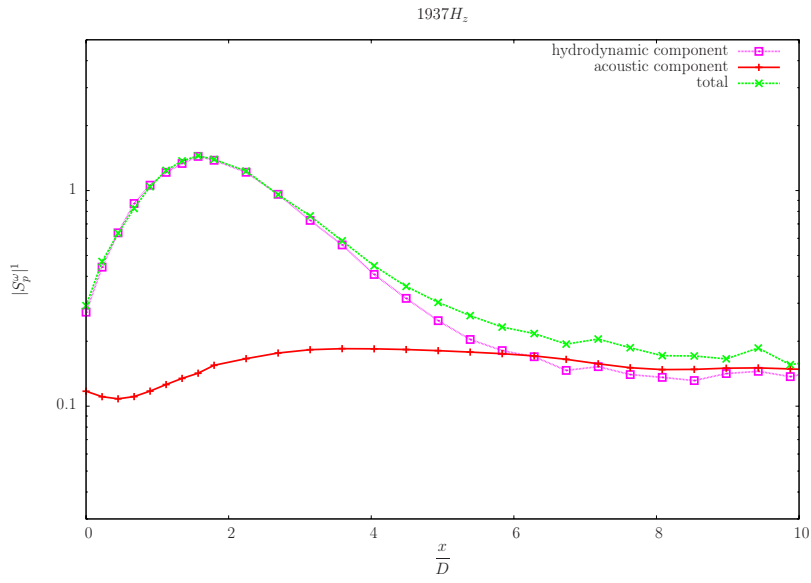

(c)

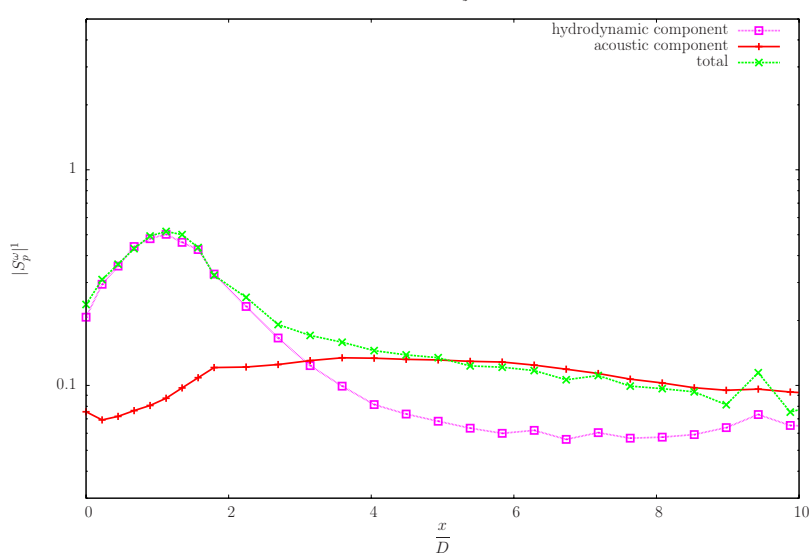

(e)

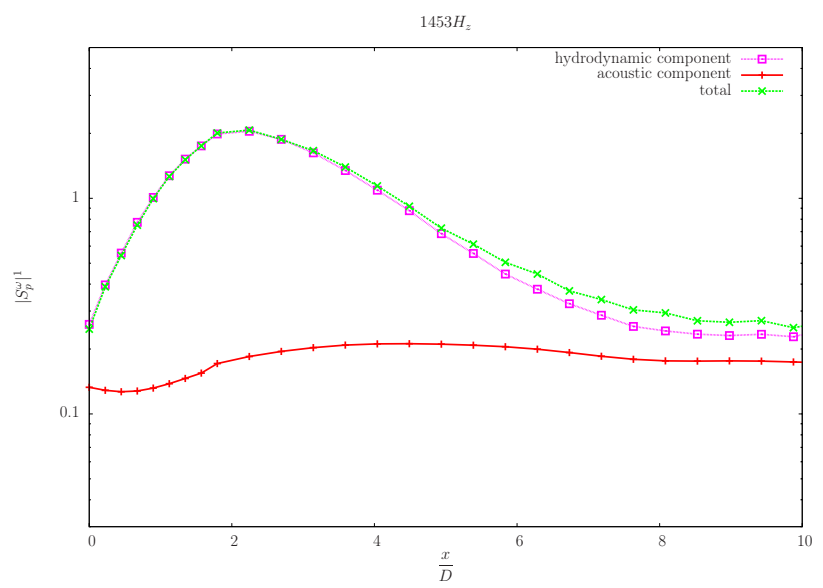

(b)

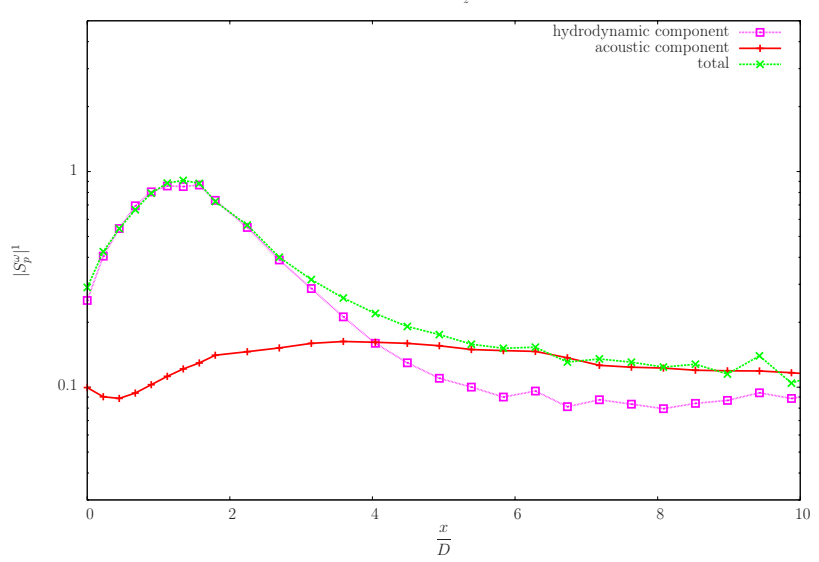

(d)

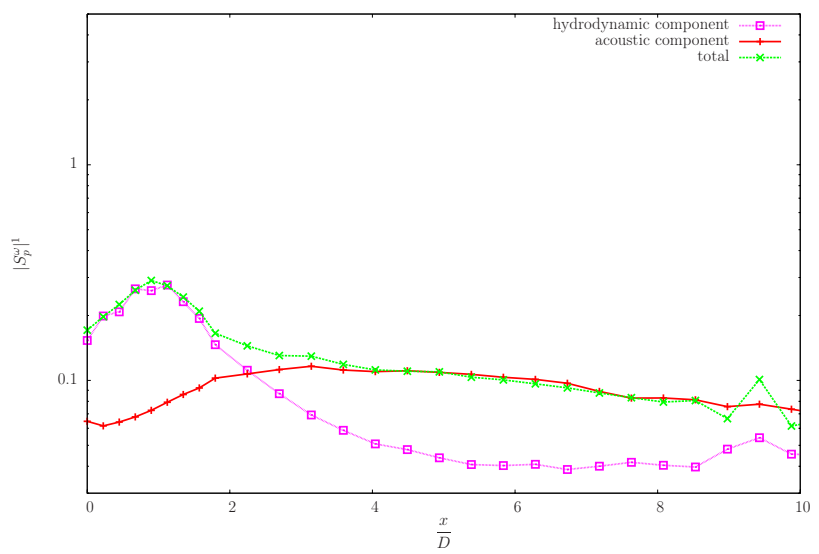

(f)

Figure 4. (a) Wavenumber-frequency spectrum; (b)-(f) axial rms distribution on line array for spectral components: $1453 \mathrm{~Hz}, 1937 \mathrm{~Hz}, 2421 \mathrm{~Hz}, 2906 \mathrm{~Hz}$ and $3390 \mathrm{~Hz}$; Green lines show unfiltered levels; pink and red lines show hydrodynamic and acoustic levels, respectively. 


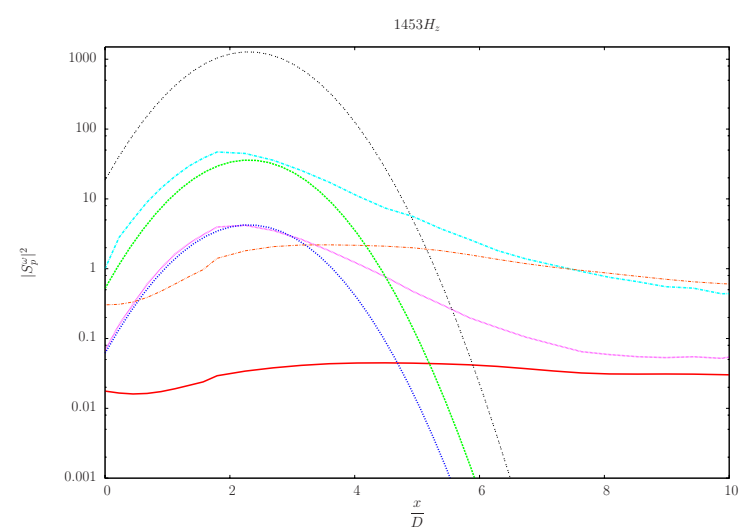

(a)

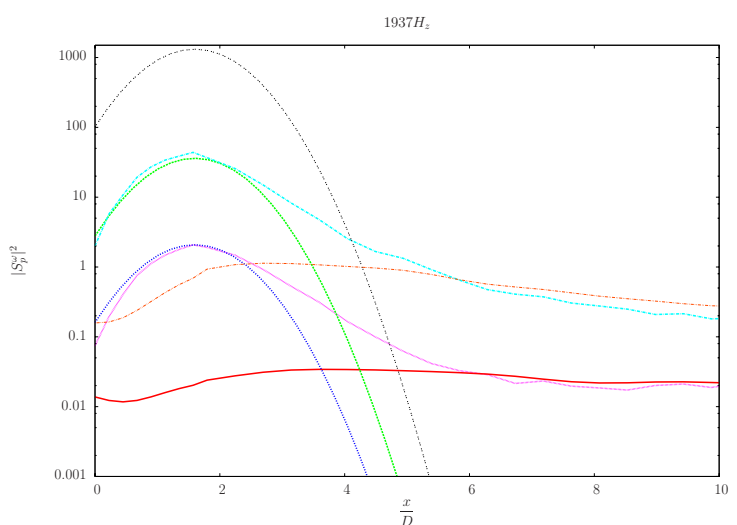

(b)

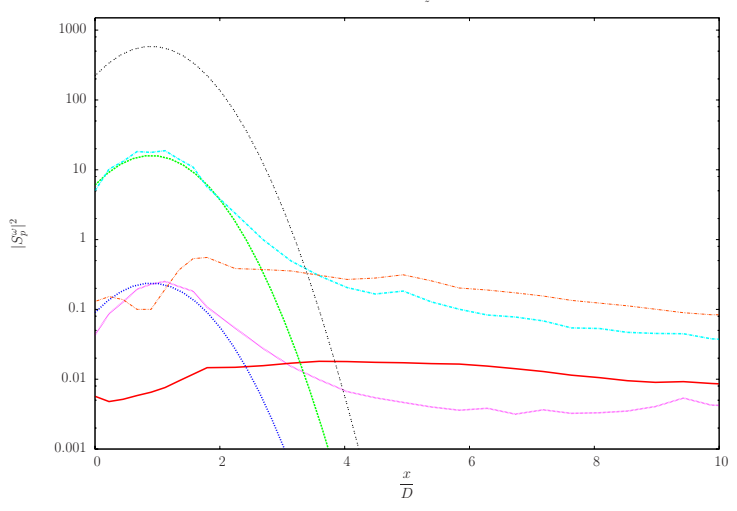

(d)

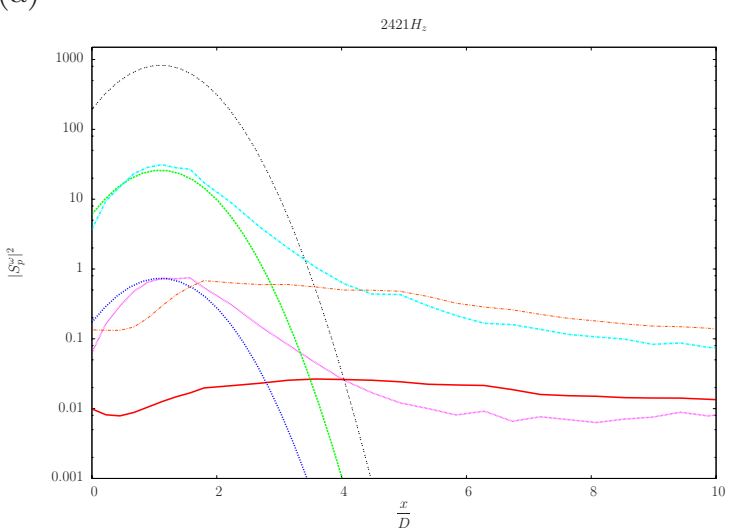

(c)

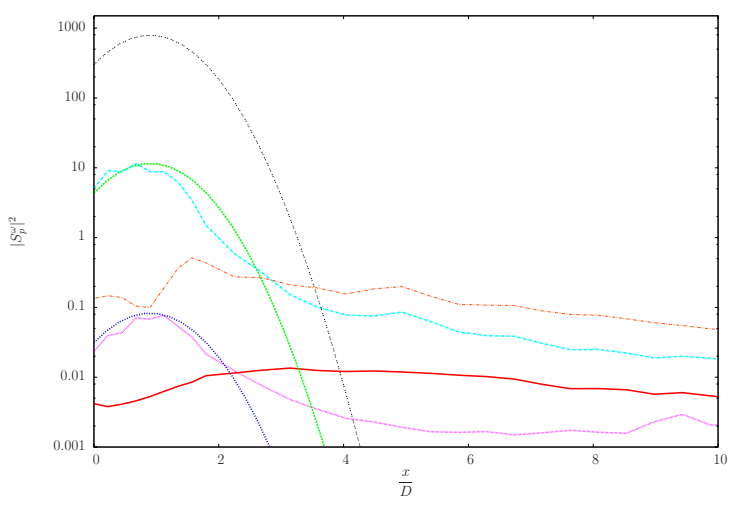

(e)

Figure 5. Hydrodynamic and acoustic energy distributions, extrapolated to give level at the shear-layer axis; (a)-(e) show results for the five frequencies presented in figure 4; hydrodynamic component extrapolated using $r^{-1} \exp \left(-r \sqrt{k^{2}-\omega^{2} / c^{2}}\right) ;^{47}$ acoustic levels extrapolated using $1 / r^{2}$ decay. Pink and light-blue: hydrodynamic component from measurements at two different radial positions; dark-blue and green: levels given by extrapolation; red: acoustic level from measurement; black and orange: hydrodynamic and acoustic levels extrapolated to the the shear-layer axis. 
The wave-envelope shape is then adapted, using the vorticity thickness measured at the location of the peak rms level for that frequency (obtained from figure 5 for example; similarity variables, identified by Tinney \& Jordan, ${ }^{48}$ justify this kind of scaling of the correlation shape). Characteristic wavenumbers and convection velocities are similarly obtained from the experimental data. A sound-production problem is then solved using the linear model presented in the previous section.

\section{Conclusion}

A model problem is used to demonstrate that interference nodes, first identified in the near pressure field of a high Reynolds number turbulent jet by Coiffet et al., ${ }^{7}$ and taken as evidence of a quasi-irrotational, linear, wavy-wall-like sound-source mechanism, do indeed occur in the model, potential-flow problem studied. The interference occurs when convective, reactive pressure fluctuations interfere destructively with propagative, resistive perturbations at coordinates where the reactive and propagative amplitudes and phases are suitably matched.

Experimental nearfield data is then studied. The measured pressure fluctuations are filtered in order to separate hydrodynamic and acoustic components. The characteristics of these are then examined at a selection of frequencies representative of the flow dynamics between the exit plane and the end of the potential core. The result shows hydrodynamic energy distributions, the peak locations of which are a function of frequency (higher frequencies are closer to the exit plane), and acoustic distributions whose peak is always found just downstream of the hydrodynamic distribution. This result is in qualitative agreement with the model problem studied in the first part of the paper. The hydrodynamic and acoustic signatures are then extrapolated in order to obtain representative values on the shear layer axis. The extrapolated hydrodynamic data is used to calibrate analytical models which are currently being evaluated in order to obtain quantitative comparisons with the experiment.

\section{Acknowledgments}

The authors gratefully acknowledge the Agence Nationale de la Recherche (ANR-05-BLAN-0208-02 program) and the Centre Nationale d'Études Spatiales for support. 


\section{References}

1984.

${ }^{1}$ H. Arbey and J.E. Ffowcs-Williams. Active cancellation of pure tones in an excited jet. J. Fluid Mech., 149:445-454, 1977.

${ }^{2}$ R.R. Armstrong, A. Michalke, and H. Fuchs. Coherent structures in jet turbulence and noise. AIAA Journal, 15(7),

${ }^{3}$ R. E. A. Arndt, D. F. Long, and M. N. Glauser. The proper orthogonal decomposition of pressure fluctuations surrounding a turbulent jet. J. Fluid Mech., 340:1-33, 1997.

${ }^{4}$ S. Barre, V. Fleury, C. Bogey, C. Bailly, and D. Juve. Experimental study of the properties of near-field and far-field jet noise. AIAA 06-2649, May 2006.

${ }^{5}$ J. Bridges and F. Hussain. Direct evaluation of aeroacoustic theory in a jet. J. Fluid Mech., 240:469-501, 1992.

${ }^{6}$ Y.Y. Chan. Physics of Fluids, 17(9):1667-1670, 1974.

${ }^{7}$ F. Coiffet, P. Jordan, J. Delville, and Y. Gervais. Coherent structures in subsonic jets: a quasi-irrotational source mechanism? International Journal of Aeroacoustics, 5:1-24, 2006.

${ }^{8}$ T. Colonius, S.K. Lele, and P. Moin. Sound generation in a mixing-layer. J. Fluid Mech., 330:375-409, 1997.

${ }^{9}$ D. G. Crighton and P. Huerre. Shear-layer pressure fluctuations and superdirective acoustic sources. J. Fluid Mech., 220:355-368, 1990.

${ }^{10}$ N. Prodi D. Stanzial and G. Schiffrer. Reactive acoustic intensity for general fields and energy polarization. Journal of Acoustical Society of America, 99:1868-1876, 1996.

${ }^{11}$ S. Le Dizs and C. Millet. Acoustic near field of a transonic instability wave packet. J. Fluid Mech., 577:1-23, 2007.

12 J.E. Ffowcs-Williams and A.J. Kempton. The noise from the large scale structure of a jet. J. Fluid Mech., 84:673-694, 1978.

${ }^{13}$ V. Fleury, C. Bailly, and D. Juv. Shear-layer acoustic radiation in an excited subsonic jet: experimental study. C.R. Mcanique, 333:746-753, 2005.

${ }^{14}$ H.V. Fuchs and U. Michel. Experimental evidence of turbulent source coherence affecting jet noise. AIAA Journal, $16: 871-872,1978$.

${ }^{15}$ A. Ghosh, J. Bridges, and F. Hussain. Instantaneous directivity in jet noise by multi-pole decomposition. Trans. A.SM.E., $117,1995$.

${ }^{16}$ A. Guitton, P. Jordan, E. Laurendeau, and J. Delville. Velocity dependence of the near pressure field of subsonic jets. AIAA-2007-3661, May 2007. 2007.

${ }^{17}$ A. Guitton, C.E. Tinney, P. Jordan, and J. Delville. Measurements in a co-axial subsonic jet. AIAA-2007-15, January

${ }^{18}$ W.L. Howes. Distribution of time-averaged pressure fluctuations along the boundary of a round subsonic jet. Technical report, NASA Technical note D-468, 1960.

${ }^{19}$ D. Juv, M. Sunyach, and G. Comte-Bellot. Filtered azimuthal correlations in the acoustic farfield of a subsonic jet. AIAA Journal, 17(1), 1979.

${ }^{20}$ D. Juv, M. Sunyach, and G. Comte-Bellot. Intermittency of the noise emission in subsonic cold jets. Journal of Sound and Vibration, 71(3):319-332, 1980.

${ }^{21}$ D. N. Keast and G. Maidanik. Studies in the near field noise properties of a small air jet. Technical report, Bolt, Beranek and Newman, Report 1272, 1966.

${ }^{22}$ F. Kerherve, A. Guitton, P. Jordan, J. Delville, V. fortune, Y. Gervais, and C. Tinney. Identifying the dynamics underlying the large-scale and fine-scale similarity spectra. AIAA-2008-3027, 2008.

${ }^{23}$ V. Kibens. Discrete noise spectrum generated by an acoustically excited jet. AIAA Journal, 18(4)(79-0592R), 1980.

${ }^{24}$ V.F. Kopiev and S.A. Chernyshev. Vortex ring eigen-oscillations as a source of sound. J. Fluid Mech., 341:19-57, 1997.

${ }^{25}$ V.F. Kopiev, M. Zaitsev, and S.A. Chernyshev. Comparison of vortex ring noise theory with excited jet acoustic measurements. 10th CEAS-ASC Workshop, 2006.

${ }^{26}$ V.F. Kopiev, M. Zaitsev, S.A. Chernyshev, and A.N. Kotova. The role of large-scale vortex in a turbulent jet noise. AIAA Paper 99-1839, 1999.

${ }^{27}$ J. Laufer and T. C. Yen. Noise generation by a low-mach-number jet. J. Fluid Mech., 134:1-31, 1983.

${ }^{28}$ H.K. Lee and H.S. Ribner. Direct correlation of noise and flow of a jet. Journal of Acoustical Society of America, 52(5), 1952.

${ }^{29}$ L. Maestrello. Statistical properties of the sound and source fields of an axisymmetric jet. AIAA Paper 77-1267, 1977.

${ }^{30}$ R. Mankbadi and J.T.C. Liu. Sound generated aerodynamically revisited: large-scale structures in a turbulent jet as a source of sound. Proc. R. Soc. London Ser. A, 311:183-217, 1984.

${ }^{31}$ M.H. Mayes, W.E. Lanford, and H.H. Hubbard. Near-field and far-field noise surveys of solid-fuel rocket engines for a range of nozzle exit pressures. Technical report, NASA Technical note D-21, 1959.

${ }^{32}$ A. Michalke and H.V. Fuchs. On turbulence and noise of an axisymmetric shear layer. J. Fluid Mech., 70(1):179-205, 1975.

${ }^{33}$ B.E. Mitchell, S.K. Lele SK, and P. Moin. Direct computation of the sound from a compressible co-rotatin vortex pair. J. Fluid Mech., 285:181-202, 1995.

${ }^{34}$ E. Mollo-Christensen. Measurements of near field pressure of subsonic jets. Technical report, NATO A.G.A.R.D. Report 449, 1963.

${ }^{35}$ C.J. Moore. The role of shear-layer instability waves in jet exhaust noise. J. Fluid Mech., 80(2):321-367, 1977.

${ }^{36}$ G.L. Morrison and D.K. McLaughlin. Noise generation by instabilities in low reynolds number supersonic jets. Journal of Sound and Vibration, 65:177-191, 1979. 
${ }^{37}$ C.L. Morfey N.D. Sandham and Z.W. Hu. Sound radiation from exponentially growing and decaying surface waves. Journal of Sound and Vibration, 294:355-361, 2006. 1967.

${ }^{38} \mathrm{~J}$.B. Ollerhead. On the prediction of the near field noise of supersonic jets. Technical report, NASA Report CR-857,

${ }^{39}$ C. Picard and J. Delville. Pressure velocity coupling in a subsonic round jet. Heat and Fluid Flow, 21:359-364, 2000.

${ }^{40}$ N. Reba, S. Narayanan, T. Colonius, and T. Suzuki. Modelling jet noise from organised structures using near-field hydrodynamic pressure. AIAA-2005-3093, May 2005.

${ }^{41} \mathrm{~F}$. Ricaud. Etude de l'identification des sources acoustiques a partir du couplage de la pression en champ proche et de l'organisation instantanee de la zone de melange de jet. PhD thesis, Universite de Poitiers, Poitiers,FR, 2003.

${ }^{42}$ N.D. Sandham, C.L. Morfey, and Z. Hu. Nonlinear mechanisms of sound generation in a perturbed parallel jet flow. J. Fluid Mech., 565:1-23, 2005.

${ }^{43}$ M. Schaffar and J.P. Hancy (1982). Investigation of the noise emitting zones of a cold jet via causality correlations. J. Sound and Vibration, 81(3):377-391, 1982.

${ }^{44}$ T.D. Scharton and P.H. White. Simple pressure source model of jet noise. Journal of Acoustical Society of America, $52(1), 1972$.

${ }^{45}$ J.M. Seiner. The distribution of jet source strength intensity by means of a direct correlation technique. PhD thesis, Pennsylvania State University, Pennsylvania, 1974.

${ }^{46}$ T.E. Siddon and R. Rackl. Cross-correlation analysis of flow noise with fluid dilatations as source fluctuation. Journal of Acoustical Society of America, 51(96A), 1972.

${ }^{47} \mathrm{~T}$. Suzuki and T. Colonius. Instability waves in a subsonic round jet detected using a near-field phased microphone array. J. Fluid Mech., 565:197-226, 2006.

${ }^{48}$ C.E. Tinney and P. Jordan. A study in the near pressure field of co-axial subsonic jets. In press for JFM, 2008.

${ }^{49}$ C.E. Tinney, P. Jordan, A. Hall, J. Delville, and M.N. Glauser. A time-resolved estimate of the turbulence and sound source mechanisms in a subsonic jet flow. Journal of Turbulence, 8(7):1-20, 2007. 